\title{
TRAFFIC INCIDENT MANAGEMENT IN THE PRESENCE OF HAZARDS
}

\author{
Zhiyong Wang ${ }^{\mathrm{a}, *}$, Sisi Zlatanova ${ }^{\mathrm{a}}$, John Steenbruggen ${ }^{\mathrm{b}}$ \\ ${ }^{a}$ Delft University of Technology, Dept. of Urbanism, Julianalaan 134, Delft, NL - wzy19840102@gmail.com, S.Zlatanova@tudelft.nl \\ ${ }^{b}$ VU University, Dept. of Spatial Economics, De Boelelaan 1105, 1081 HV Amsterdam, NL, john.steenbruggen@ rws.nl
}

KEY WORDS: Incident management, Evacuation, hazards, Navigation

\begin{abstract}
:
Traffic incidents can result in different kinds of hazards (e.g., plumes) that influence the status of road networks, therefore there is a great need for incident management in the presence of the hazards. When incidents occur, the created hazards not only affect the normal road users (make them detour or blocked), but also influence the movement of first responders. Traffic managers, who are responsible for maintaining the road safety and traffic stability, should carry out quick and effective measures to manage the incidents. In this paper, we present four issues to help people better understand the situations that could occur in the management of incidents with hazards: 1). Evacuation in the presence of hazards; 2). 3D incident management; 3). Navigation support for first responders; 4). Navigation support for road users. To address these issues, we propose a solution which combines agent technology, geo-database, hazard simulation, and traffic simulation. Further research would be needed to investigate the potentials of the proposed solution in real applications.
\end{abstract}

\section{INTRODUCTION}

Traffic incidents have a vast effect on the present societies, causing economic loss as well as causalities (Steenbruggen et al., 2014). In the event of major incidents (e.g., with toxic substance), not only the road users but also the surrounding communities can be affected. This poses a large set of challenges, which requires a successful traffic incident management as well as innovative technologies for coping the incidents.

Major incidents can create all kinds of hazards that influence road users on highways as well as people who live nearby. For example, when a truck that transports toxic chemicals has a traffic incident, the dangerous goods can be released and form obstacles that move around the incident location. They have a direct impact on the people who are close to the incident location, blocking their routes. Furthermore, there is indirect influence on other road users who are traveling in the affected road network. The roads they plan to use would be unavailable because of the moving obstacles and new routes should be provided to them considering the blocked roads. Besides, people who live in the surrounded area are also vulnerable to these accidents. Especially when the accidents happen close to communities with dense population (e.g., hospitals, schools, shopping malls), the released dangerous materials can threaten the safety of the surrounding communities and these people should be evacuated as soon as possible.

Incident management (IM) plays a crucial role in minimizing the negative impact of incidents. It includes a set of measures, such as clearing the blocked roads for traffics, evacuating the victims, controlling the damage, ensuring safety for emergency work and road users (Steenbruggen et al., 2013). In Europe, traffic incident management involves multiple public agencies (e.g., road authorities) and private-sector partners. Although there is a great variety in the national road administrations, in all European countries the same services (police, fire brigade, and ambulance services) are provided in IM (Steenbruggen and Nijkamp, 2012). These involved agencies apply the IM operations, coordinate their operations and activities, and help to prevent incidents and

${ }^{*}$ Corresponding author rescue victims. Traffic incident management is a complex process that can be broken into a set of tasks performed by the agencies at different levels (Zlatanova, 2010). At strategic level, traffic managers are responsible for making rescue plans, collect and distribute information, and give instructions to first responders as well as affected people. At the operational level, first responders should go to the incident place to perform their rescue works, and carry out measures to prevent further damage.

To help emergency agencies carry out the IM process, there is a great need for real-time (spatial) information and supporting information systems. The access to relevant data or expertise is essential for an effective crisis response (Hale, 1997). Recent studies show that information (sharing), communication, and coordination are the main bottlenecks for effective cooperation between emergency services (Comfort et al., 2004; Chen et al., 2008). Although currently used risk maps can give an overview of potential risks in incident places, dealing with the incidents with dangerous substances requires a higher level of preparedness and good knowledge of situations. Not only real-time information of the current environment is required, but also predicted information of future situations, e.g., predicted traffic flows, the movement of hazards is needed. Such information is quite important for successful traffic management, assisting traffic managers to develop strategic plans and helping road users to adjust their routes to their destinations. New innovative technology and systems should be developed to deliver both real-time and predicted information. This requires a multidiscipline approach, which combines the results from a wide of range of fields, e.g., fire simulation (Moreno et al., 2014; $\mathrm{Hu}, 2011$; Xue et al., 2012), plume modeling (Zelle et al., 2013; García-Díaz and Gozalvez-Zafrilla, 2012), and traffic predictions (Li and Chen, 2014; Fei et al., 2013).

In this paper, we identify and present four issues in the management of traffic incidents that involve hazards. For each issue, we describe related scenarios that could occur during the incidents, and give our analysis. Besides, potential research problems that need to be investigated are also defined in the paper. We don't claim that this paper covers all the issues in managing incidents with the hazards. But we believe that our descriptions and analysis of these issues would help people understand the characteristics and differences of the relevant 
problems, and provides insights on solutions to these issues. The remainder of the paper is organized as follows. In Section 2, we present the four issues during the management of incidents with the hazards. To address these issues, in Section 3, we propose our approach, which combines Geographic Information System (GIS), hazard modeling, and traffic simulation. This paper is concluded in Section 4.

\section{ISSUES}

In this section, we give a list of issues that need to be considered for the management of incidents with hazards.

\subsection{Issue 1: Evacuation in the presence of hazards}

Maintaining the safety for road users is one of the main responsibilities for road authorities in traffic management (Steenbruggen and Nijkamp, 2012). When an accident happens to a truck transporting dangerous goods, the toxic materials can be released, which results in moving obstacles. These obstacles can not only block vehicles that are passing by, but also affect the safety of road users who are close to the incident place on the highway (as shown in Figure 1). Such situations require traffic managers to carry out necessary measurement to respond effectively to the incidents. The affected people should be informed about the risk of obstacles, and be evacuated from the dangerous areas to safe place as soon as possible. Moreover, in the event of major incidents, dangerous substance on or near a highway could also have a direct impact on the safety of the people who are living in the surrounded area. In this case, the information of hazards can be distributed by traffic information services and can also be shared to citizens living next to the highway. In certain cases, these people need to be provided with evacuation routes on their smartphone. The incidents can also cause traffic disorder or disturbance, which could influence the movement of vehicles in the roads (Tu et al., 2008). On the other hand, the traffic management center can directly take actions, e.g., blocking certain roads, to control the traffic flows to facilitate the evacuation process. In the case that the incidents are close to hospitals and schools, public transportation services, e.g., buses, trams, and trains, would be needed to help evacuate the citizens. The questions that are related to this issue and need to be investigated are: 1). How can traffic incident managers evacuate the road users who are blocked by the hazards on the highways? 2). How can traffic incident managers evacuate the people who are living close to the incident place, considering the hazards and different transportation modes? 3). What kind of measures traffic incident managers should take to reduce the impact of hazards?

\subsection{Issue 2: 3D incident management}

With the development of the highway networks, more and more roads pass through cities above or under the ground, which brings the necessity of using 3D information for incident management (Zlatanova and Holweg, 2004). For example, in the event of plumes caused by a truck incident, the released hazardous materials, which have the third dimension, can spread into the built structure, having fatal effects on built environment. To help evaluate the situations, 3D representation of hazards and the highways as well as their surroundings (as presented in Figure 2) would be needed. With 3D visualization of the environment, emergency managers can clearly see which floors in the building would be affected by the hazards, and take measures accordingly, such as, informing the people at the threaten floors to get out of building first, evacuating the victims

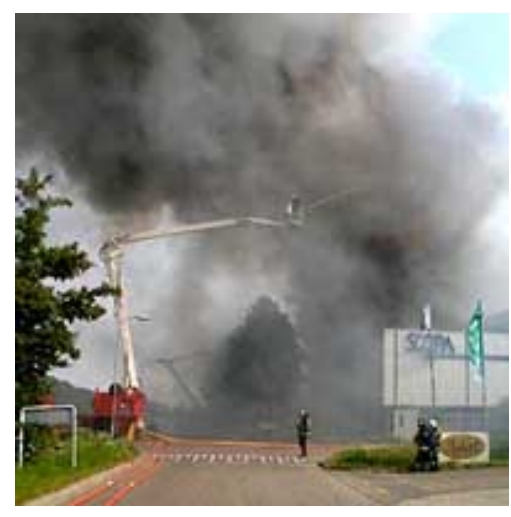

(a)

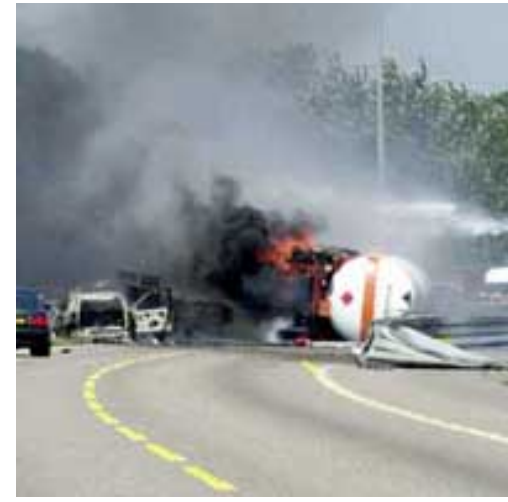

(b)

Figure 1: Traffic incidents that cause hazards.

to safe places (e.g., parking slots) inside the building. 3D models (e.g, CityGML), which represent the environment in different levels of details, can also help decision making at different levels (Kemec et al., 2009). For example, in the event of fires caused by an incident in tunnels, 3D models of the affected environment can help emergency managers at the command and control center to obtain a good overview of the situations on and under the ground, support making strategic decisions. While traditional 2D maps can only provide limited information under the ground, 3D representation and visualization can give a detailed picture of the environment, e.g., the structure of the tunnels, the vulnerable objects in the tunnels, the location and distribution of road users. This would help responders, who perform the field works, to fast and easily locate the incident and the facilities that are inside the tunnels. Some questions that are relevant to this issue are: 1). What kind of 3D models would be needed to represent the environments affected by hazards, supporting different levels of details? 2). What kind of 3D visualization methods should be used to support the emergency managers at commander and control center as well as the responders on the field? 3). What kind of 3D spatial analysis should be developed to support decision makings at different levels (operational, tactic and strategic)?

\subsection{Issue 3: Navigation support for first responders}

When an incident happen, first responders from different agencies, e.g., ambulances, fire brigade, should go to the incident place to deal with the incidents and help the victims. Multiple destinations can be involved if more incidents occur (Wang and Zlatanova, 2013b). When responders move towards their destinations, the obstacles caused by the incidents can influence the state of roads and should be taken into account the routing process (Wang and Zlatanova, 2013a; Visser, 2009; 


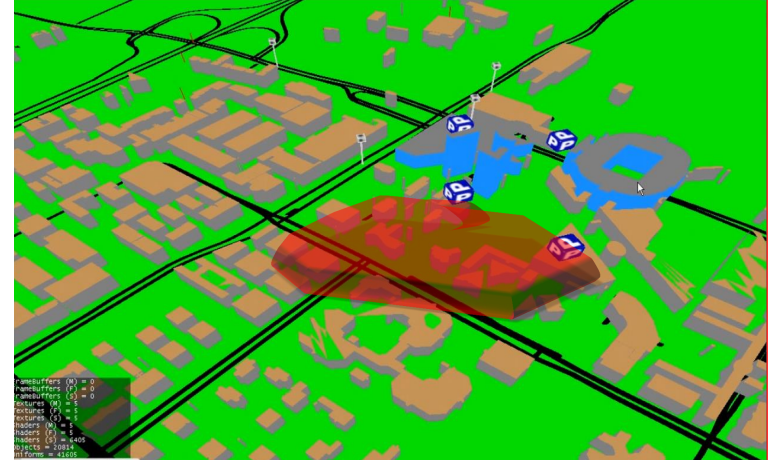

Figure 2: 3D representation of a truck incident that generates toxic plumes (in red). The black lines represent the roads. The roofs and walls of the buildings are colored in brown and grey respectively.

Nedkov and Zlatanova, 2011). Besides, the traffic flows on the highways can have an impact on the movement of the vehicles. Therefore the traffic information of the road network should also be integrated into the path planning for first responders. Another important factor that needs to be considered in the routing is the profile of responders. Because responders can have protective equipment (e.g., masks) which would allow them to go through the obstacles (e.g., plumes), as illustrated in Figure 3. Guiding the responders to go through the obstacles can greatly reduce the response time, which would facilitate the incident management process. The questions that need to be addressed are: 1 ). What kind of algorithms would be needed to deal with the traffic information of road networks to generate the safe routes for first responders? 2) How can we incorporate the profile of responders to help them go through the obstacles? 3). What kind of data models would be needed to support the integration of traffic information?

\subsection{Issue 4: Navigation support for road users}

Another important issue is the navigation aid for ordinary road users after traffic incidents occur. Because the hazards caused by the incidents could make certain roads blocked, this information should be integrated into route navigation systems to provide alternative driving routes to the vehicles on the highway. Moreover, in the case of big incidents, disorders and disturbance could happen to the traffics in the road network, resulting significant delays on some roads. This information can be distributed by traffic information services to give precise travel information to road users. To better support navigation for road users, a traffic simulation system that can integrate the real-time information would be needed to evaluate the behaviors of people and to make short-term predictions of traffics during the incidents. Figure 4 shows an example of traffic prediction results. Because re-routing road users could still cause unexpected congestion and delays, traffic management strategies would be conducted. The simulation system should also take into account the strategies and measures taken by traffic managers to forecast the possible situations. The predicted traffic information from simulations can be distributed by traffic manage services and be used by navigation systems of road users. Some questions that deserve further study are: 1). How can we navigate road users to their destinations in the presence of moving obstacles, in the meantime minimizing the possible congestion or delays. 2). What kind of traffic simulation systems should be developed to predict the traffics, taking into account the influence of hazards? 3). What kind of measures should the traffic managers take to prevent the possible congestions when they provide traffic information services to the road users?

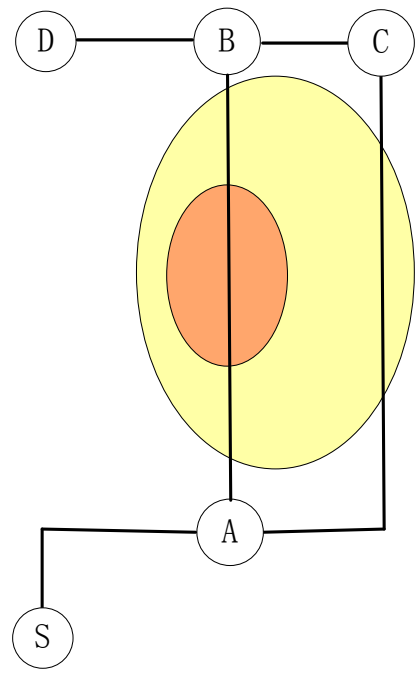

(a) A simple road network affected by smoke plumes with different concentrations of toxic materials. The concentration in the yellow zone is 0.5 parts per million; The concentration in the red zone is 20 parts per million.

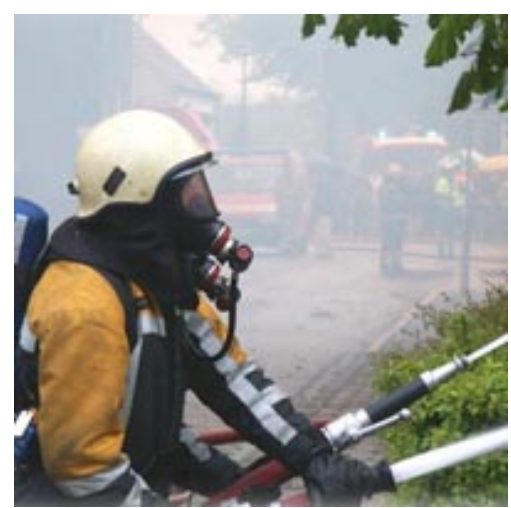

(b) A responder wearing a gas mask. We assume that the responder is allowed to stay for a certain period of time within the yellow zone with the mask, but is forbidden to enter the red zone.

Figure 3: Navigation for a responder with a gas mask.

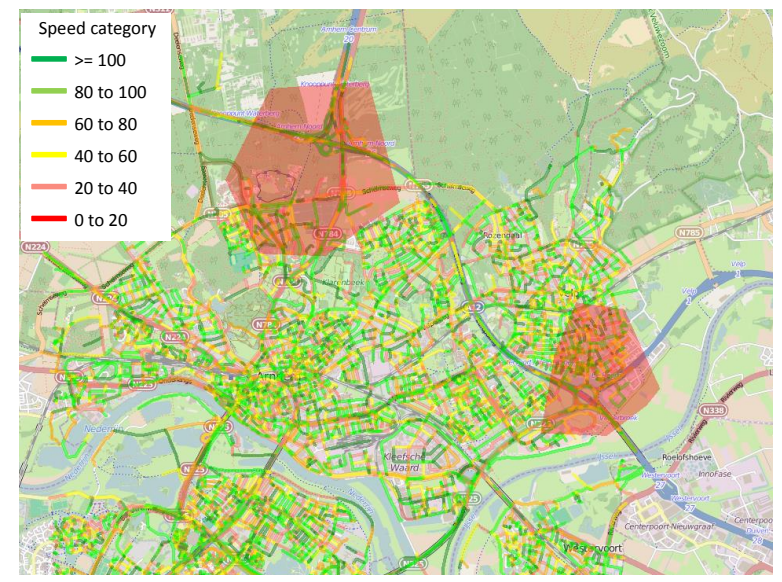

Figure 4: A snapshot of simulation results of traffic flows in a road network. The roads are colored differently based on the predicted travel speeds on the roads at a certain time point.

\section{OUR APPROACH}

To address the issues outlined in Section 2, in this paper we adapt the previous work presented in Wang et al. (2014) and 
propose an integrated framework for IM. Our framework combines agent technology, geo-database, real-time information system, hazard simulation system, and the travel time prediction system. Figure 5 shows the proposed framework. Real-time information of the environment (e.g., the speed of winds, the traffic flows) is collected by measuring devices (e.g., loop detectors, video cameras) and mobile sensors (e.g., smart phones), and is stored and distributed by a central real-time information system. The central real-time information system provides the real-time data to the traffic simulation system, which predicts the future traffic situations in transportation networks. If also feeds the hazard simulation system with the real sensor measurements needed for predictions of hazards. All the predicted information for incident management is gathered and structured in the geo-database, and is used by the multi-agent system. Various types of agents can be developed and integrated into this framework to perform different kinds of spatial processing and analysis, e.g., transforming the data into standard GIS format, calculating the routes avoiding the hazards. The agents can also be customized with special knowledge and capabilities, supporting decision making at different levels.

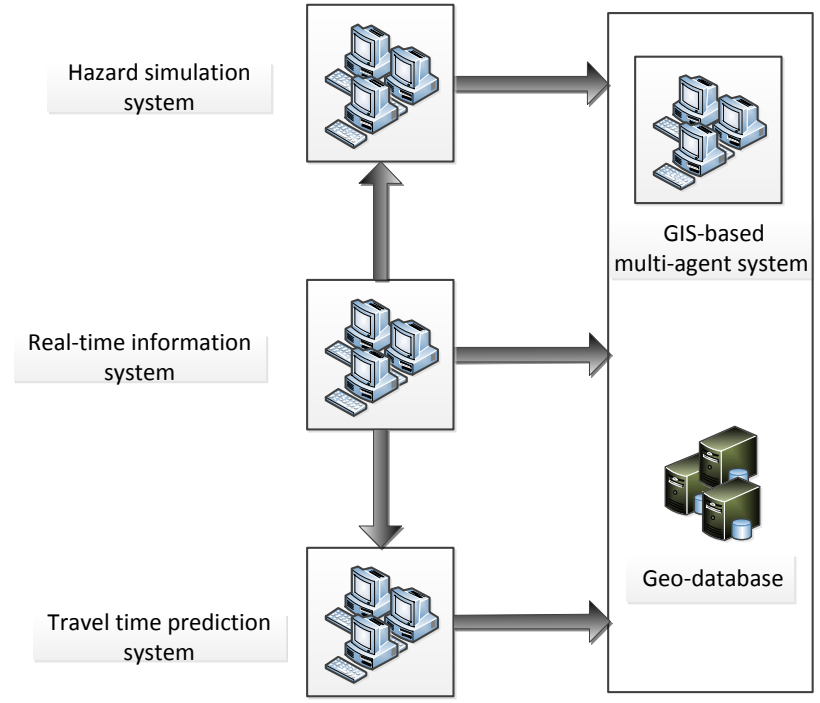

Figure 5: The proposed framework

\section{CONCLUSIONS}

This paper concentrates on the traffic management in the presence of hazards. When incidents occur, the dangerous materials (e.g., plumes) can be created and influence the status of road networks. They not only affect the safety and behaviors of road users, but also influence the movement of first responders. To help traffic incident managers to manage such incidents, in this paper we have presented four issues that need to be addressed and give our analysis on these issues. Furthermore, we have proposed an approach for dealing with these incidents, which combines agent technology, geo-database, hazard simulation and traffic simulation. Such an approach not only supports planning of incident management processes, but also allows for evaluation of strategies.

It should be noted that the handling the incidents with hazards is a complicated process, which requires research efforts in many different fields, e.g., GIS, hazard simulation, traffic modeling and simulation. The involved systems need to be inter-connected to enhance their capabilities, for example, to model behaviors of road users in the presence of hazards, the information of hazards should be integrated into the traffic simulation system to make the prediction of traffics more reliable. Besides, the presented issues in this paper are mainly spatial-related, other aspects are also open and need further investigation, for instance, the origination of efforts for incident management, standardization of processes for incident response.

\section{ACKNOWLEDGEMENTS}

This work was sponsored by Rijkswaterstaat, Ministry of Infrastructure and the Environment, The Netherlands.

\section{REFERENCES}

Chen, R., Sharman, R., Rao, H. R. and Upadhyaya, S. J., 2008. Coordination in emergency response management. Communications of the ACM 51(5), pp. 66-73.

Comfort, L. K., Ko, K. and Zagorecki, A., 2004. Coordination in rapidly evolving disaster response systems the role of information. American Behavioral Scientist 48(3), pp. 295-313.

Fei, X., Zhang, Y., Liu, K. and Guo, M., 2013. Bayesian dynamic linear model with switching for real-time short-term freeway travel time prediction with license plate recognition data. Journal of Transportation Engineering 139(11), pp. 1058-1067.

García-Díaz, J. C. and Gozalvez-Zafrilla, J., 2012. Uncertainty and sensitive analysis of environmental model for risk assessments: An industrial case study. Reliability Engineering \& System Safety 107, pp. 16-22.

Hale, J., 1997. A layered communication architecture for the support of crisis response. Journal of Management Information Systems pp. 235-255.

Hu, X., 2011. Dynamic Data Driven Simulation. SCS M\& S Magazine pp. 662-669.

Kemec, S., Duzgun, H. S. and Zlatanova, S., 2009. A conceptual framework for $3 \mathrm{~d}$ visualization to support urban disaster management. In: Proceedings of the Joint Symposium of ICA WG on CEWaCM and JBGIS G14DM, 268-278.(2009), International Cartographic Association, ICA/ISPRS.

Li, C. S. and Chen, M. C., 2014. A data mining based approach for travel time prediction in freeway with non-recurrent congestion. Neurocomputing 133, pp. 74-83.

Moreno, A., Posada, J., Segura, Á., Arbelaiz, A. and GarcíaAlonso, A., 2014. Interactive fire spread simulations with extinguishment support for virtual reality training tools. Fire Safety Journal 64, pp. 48-60.

Nedkov, S. and Zlatanova, S., 2011. Enabling obstacle avoidance for Google maps' navigation service. In: Altan, Backhause, Boccardo \& Zlatanova (Eds.), International Archives ISPRS XXXVIII, 7th, Gi4DM, Anltalya, Turkey.

Steenbruggen, J. and Nijkamp, P., 2012. Traffic incident management in europe-guide for best practice. on Geoinformation for Disaster Management-Best Practices pp. 9-34.

Steenbruggen, J., Borzacchiello, M. T., Nijkamp, P. and Scholten, H., 2013. Data from telecommunication networks for incident management: An exploratory review on transport safety and security. Transport policy 28, pp. 86-102. 
Steenbruggen, J., Nijkamp, P. and van der Vlist, M., 2014. Urban traffic incident management in a digital society: An actornetwork approach in information technology use in urban europe. Technological Forecasting and Social Change 89, pp. 245-261.

Tu, H., Van Lint, H. and Van Zuylen, H., 2008. The effects of traffic accidents on travel time reliability. In: Intelligent Transportation Systems, 2008. ITSC 2008. 11th International IEEE Conference on, IEEE, pp. 79-84.

Visser, I., 2009. Route determination in disaster areas. Master's thesis, GIMA MSc programme (Utrecht University, TU Delft, Wageningen University, ITC).

Wang, Z. and Zlatanova, S., 2013a. An A*-based search approach for navigation among moving obstacles. In: S. Zlatanova, R. Peters, A. Dilo and H. Scholten (eds), Intelligent Systems for Crisis Management, Springer, pp. 17-30.

Wang, Z. and Zlatanova, S., 2013b. Taxonomy of navigation for first responders. In: J. M. Krisp (ed.), Progress in Location-Based Services, Springer, pp. 297-315.

Wang, Z., Zlatanova, S., Moreno, A., van Oosterom, P. and Toro, C., 2014. A data model for route planning in the case of forest fires. Computers \& Geosciences 68, pp. 1-10.

Xue, H., Gu, F. and Hu, X., 2012. Data assimilation using sequential monte carlo methods in wildfire spread simulation. ACM Transactions on Modeling and Computer Simulation (TOMACS) 22(4), pp. 23.

Zelle, H., Wisse, E., Mika, Á. and van Tilburg, T., 2013. Smoke plume modeling in crisis management. In: Intelligent Systems for Crisis Management, Springer, pp. 51-66.

Zlatanova, S., 2010. Formal modeling of tasks to support search of geo-information in emergency response. In: International symposium climate change and disaster management. GIS Development Pvt. Ltd., Noida, pp. 26-36.

Zlatanova, S. and Holweg, D., 2004. 3D Geo-information in emergency response: a framework. In: Proceedings of the 4th International Symposium on Mobile Mapping Technology (MMT'2004), March, pp. 29-31. 